\title{
The Expected Lifetime of an Extraction Project
}

Evatt, G. W. and Johnson, P. V. and Duck, P. W. and Howell, S. D. and Moriarty, J.

2011

MIMS EPrint: 2011.18

Manchester Institute for Mathematical Sciences

School of Mathematics

The University of Manchester

\footnotetext{
Reports available from: http://eprints.maths.manchester.ac.uk/

And by contacting: The MIMS Secretary

School of Mathematics

The University of Manchester

Manchester, M13 9PL, UK
} 


\title{
The Expected Lifetime of an Extraction Project
}

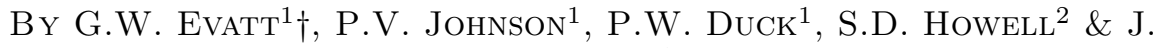 \\ MORIARTY ${ }^{1}$ \\ ${ }^{1}$ School of Mathematics, University of Manchester, Oxford Road, Manchester M13 \\ $9 P L, U K$ \\ ${ }^{2}$ Manchester Business School, University of Manchester, Booth Street West, \\ Manchester M15 6PB, UK.
}

When a mining company begins extraction from a finite resource, it does so in the presence of numerous uncertainties. One key uncertainty is the future price of the commodity being extracted, since a large enough drop in price can make a resource no longer cost-effective to extract, resulting in the mine being closed down. By specifying a stochastic price process, and implementing a financial-type model which leads to the use of partial differential equations, this paper creates the framework for efficiently capturing the probability of a mine remaining open throughout its planned extraction period, and derives the associated expected lifetime of extraction. An approximation to the abandonment price is described, which enables a closed-form solution to be derived for the probability of operational success and expected lifetime. This approximation compares well with the full solution obtained using a semi-Lagrangian numerical technique.

Keywords: Partial Differential Equations, Finite Resource Valuations, Feynman-Kac, Real Options.

\section{Introduction}

To make effective plans for a commercial venture, a company is often required to consider the associated probability of project completion. This is particularly relevant to the mining industry, where large up-front capital investments are required. When a mining company takes the decision to extract from a reserve, it does so in the presence of numerous uncertainties, which will be present for (potentially) decades to come. These uncertainties can come from many sources, such as political risk and labour market changes, however, the key financial uncertainties are captured by the commodity price variations and errors in the estimated ore-grade, since it is the product of these two quantities that generates the cash in-flows for a mining operation. Such fluctuations in the underlying commodity price can help produce large profits for some mines, whilst at the other extreme they can cause others to close down and abandon operation altogether, since the extraction project will no longer be cost effective.

The option to abandon a project has been considered in the context of numerous commercial ventures such as the optimal management of shipping operations, machine plants and investment projects, to name but a few (Trigeorgis 1993; Dixit \&

$\dagger$ Author for correspondence: geoffrey.evatt@mbs.ac.uk 
Pindyck 1994). Whilst the application of an abandonment option within mine valuation was first analysed by Brennan \& Schwartz (1985), the associated probability of project completion, or expected duration (lifetime) of extraction, has not been previously considered. However, if known, the probability of project completion can be of great use to a company, as it is a quantitative measure of a project's risk, and easily interpreted by non-specialists. Why such probabilistic stopping problems have been considered in many fields, such as physics, biology and finance, but not as yet within mining, is clear: it is because the added difficulty of including the extra dimension of reserve size has greatly hindered making representative mine valuations (MacCarthy \& Monkhouse 2002). However recent advances in the application of numerical techniques by Thompson et al. (2004) and Chen \& Forsyth $(2007,2009)$ now make possible such contingent claims valuations, and allow us to consider problems such as those posed in this paper.

Mine valuations in the presence of price uncertainty are typically made using a contingent claims approach, as first studied in a simplified mine by Brennan \& Schwartz (1985). In this earlier work, the authors allowed the operational mine to either be abandoned or mothballed, depending upon the underlying price. It is not uncommon to consider additional financial uncertainties (such as interest rate) in other, more standard, contingent claims valuations, such as forward contracts (Hilliard \& Reis 1998; Gibson \& Schwartz 1990). However these additional factors are not such a pressing concern in a finite resource valuation, as the next most important uncertainty (after price) is the estimated ore-grade. The inclusion of ore-grade uncertainty has been considered by Menabe et al. (2004), Jewbali \& Dimitrakopoulos (2009) and Martinez (2009), amongst others, who specified the ore-grade to behave in a Gaussian fashion, and solved their models using a MonteCarlo simulation approach. By using such an approach, valuations often take a long time to be computed (Caccetta \& Hill 2003), with model sensitivities being difficult to extract. In addition, no method to determine the parameter estimates surrounding the uncertainty is explicitly given. Evatt et al. (2010) showed how grade uncertainty could be addressed within an efficient partial differential equation (PDE) framework, providing estimated parameter values for each individual dataset from a mine, thus allowing accurate and representative solutions to be derived quickly. Whilst this work created a readily usable framework for valuation under both price and ore-grade uncertainties, the overall effect of including ore-grade uncertainty proved to be minor in comparison to that of the price. As such, the present paper will only consider price uncertainty.

In $\S 2$ we show how a general PDE framework can be created for generating the probability of project completion, the expected lifetime and the mine valuation of an optimally managed mine. Using the valuation equation, we then show in $\S 3$ how the optimal position of abandonment can be derived and how a constant approximation to it can be easily calculated. With this approximation, $\S 4$ shows how a convenient closed-form solution for the probability of project completion and expected lifetime can be constructed. The semi-Lagrangian numerical scheme used to solve the full problem is detailed in $\S 5$. With our model built and solution method constructed, we apply it to a real mine (which is planned to last no more than 15.3 years) in $\S 6$, to see how the probability of closure varies during its extraction schedule. Our conclusions are presented in $\S 7$. 


\section{Modified Feynman-Kac equation}

The derivation of the probability of project completion (which we shall now refer to as 'completion') and the expected lifetime are closely linked to the valuation of the mine, since they share the same underlying uncertainty. By writing $t$ for (chronological) time, and $S_{t}, Q_{t}$ for the commodity price and resource size respectively at time $t$, the valuation is a function of $t, S_{t}$ and $Q_{t}$. As explained in $\S 1$, we do not treat the ore-grade, $G$, as a state variable for these valuations. Instead we regard it as an input function of resource size, which has been calculated (estimated) from geological surveying (e.g. drilling). There are two standard methods we might use to arrive at the core equation which governs the mine valuation. However, one of these methods is perhaps more naturally extended to deriving the probability of completion and the expected duration of extraction. The first method is the contingent claims approach, where hedging arguments are used to create a riskless portfolio which contains the mine and is short (owes) a number of commodity contracts. This type of analysis has been extensively covered, e.g. Black \& Scholes (1973), Black (1976) and Heath et al. (1992). Indeed, when mine valuations in the presence of uncertainty are considered, a contingent claims approach is typically used, see for example Brennan \& Schwartz (1985), Cherian et al. (2000) and Evatt et al. (2010). The second approach uses hedging arguments to specify the stochastic dynamics of the risk-adjusted commodity price process, and then applies probabilistic methods to obtain a modified Feynman-Kac equation, see for example Øksendal (2003). Whilst either method may be used to value the mine, we believe that the application of the modified Feynman-Kac equation in this context is novel. Since this probabilistic method also provides a convenient framework in which we may derive the probability of completion and expected lifetime, we use this method within this paper.

We first develop a general PDE which governs a quantity $u$, within a solution domain $H$. This PDE can subsequently be used to describe the mine valuation $V\left(S_{t}, Q_{t}, t\right)$, the probability of completion $P\left(S_{t}, Q_{t}, t\right)$, and the expected lifetime of extraction $D\left(S_{t}, Q_{t}, t\right)$, where $\left(S_{t}, Q_{t}, t\right)$ is a particular point in time of the solution domain given by $(S, Q, t)$. To achieve this we first consider a general class of expectations of the form

$$
u(x)=E_{x}\left[e^{-c \nu} f\left(X_{\nu}\right)+\int_{0}^{\nu} e^{-c u} g\left(X_{u}\right) d u\right],
$$

where $f$ and $g$ are functions to be defined, $c$ is a constant, $X_{t}$ is an Ito diffusion in $\mathbf{R}^{n}$ such that

$$
d X=b(X) d t+\sigma(X) d B
$$

where $B$ is a standard Wiener process in $\mathbf{R}^{n}$, with drift $b \in \mathbf{R}^{n}$ and volatility $\sigma \in \mathbf{R}^{n \times n}, \nu$ is the (random) first time that $X_{t}$ exits the solution domain $H$, and $E_{x}$ denotes the expected value when $X_{0}=x \in \mathbf{R}^{\mathbf{n}}$. We may now use a generalisation of the Feynman-Kac formula to link this stochastic process construction to PDEs. For a full derivation we refer to Øksendal (2003), which shows that the above equation is the solution to the problem

$$
\begin{aligned}
& L u(x)-c u(x)=-g(x) \quad \text { in } H \\
& \lim _{x \rightarrow y} u(x)=f(y) \text { for } y \in \partial H
\end{aligned}
$$


where,

$$
L \equiv \sum_{i, j=1}^{n} a_{i j} \frac{\partial^{2}}{\partial x_{i} \partial x_{j}}+\sum_{i=1}^{n} b_{i} \frac{\partial}{\partial x_{i}}
$$

and $\left[a_{i j}\right]=\frac{1}{2} \sigma \sigma^{\prime}$. Although (2.3) may at first appear to be an elliptic equation, by specifying time to follow the trivial stochastic process $d t=1 . d t+0 . d B$ as part of (2.2), equation (2.3) turns into a parabolic form.

With this formulation, the expectation (2.1) is sufficiently general to represent any one of our three desired quantities $P, D, V$, if the parameter $c$ and functions $f, g$ are chosen appropriately for the problem. The parameter $c$ is the (possibly zero) constant discount rate appropriate to the problem, $f$ is the solution's boundary value, and $g$ is the instantaneous source term appropriate to the problem. In other words, the solution $u$ is the expectation of the boundary value, where $X_{t}$ exits $H$, plus accumulated source terms, under appropriate discounting.

The solution domain in which we work is parametrised by $(S, Q, t)$, and thus we work with an Ito diffusion in $\mathbf{R}^{3}$. Indeed, it is the presence of the extra dimension $Q$ that distinguishes the valuation of a mine from more classical finance problems, which are typically obtained in $(S, t)$ solution domain. How $Q$ and $t$ interact is governed by the relation

$$
d Q=-q d t
$$

where $q$ is the rate of extraction of ore-bearing material from the mine and is bounded by the physical constraint $q \in\left(0, q_{\max }\right)$. The form of $q$ will be specified by the mining operators, but can be a function of all three variables, $S, Q$ and $t$. The mining operation will cease when either the lease has reached expiry, at time $T$, or when the mine is exhausted, $Q=0$.

The uncertainty we consider within this paper is a standard price process, namely geometric Brownian motion, described by,

$$
d S=\mu S d t+\sigma S d B
$$

where $\mu$ is the percentage drift and $\sigma$ the percentage volatility of $S$. Other Ito diffusions may be chosen to model the price process, such as mean-reverting Brownian motion (which is often quite suitable for commodities, see Bessembinder et al. 1995) or those suggested by Schwartz (1997), and they may also be treated utilising the modified Feynman-Kac equation framework described here.

The boundary $\partial H$ is that described by the two planes $t=T$ and $Q=0$, corresponding respectively to expiry of the mine's lease and exhaustion of the resource, the far field condition as $S \rightarrow \infty$, and the price surface of abandonment, denoted by $S^{*}(Q, t)$. Any point upon the surface of abandonment may be regarded as the price for which the mining company is currently indifferent as to whether the mine is kept open or abandoned altogether. Our goal here is to compute the probability of the price crossing this price surface, at which moment the mining operation is abandoned. How to calculate this surface was first discussed by Brennan \& Schwartz (1985), and is described in $\S 3$.

With our particular choice of Ito diffusion $X(t)=\left(S_{t}, Q_{t}, t\right)$, the quantity $u(S, Q, t)$ of prime interest, and making the substitution $\tau=T-t$, the PDE in 
(2.3) becomes

$$
\frac{1}{2} \sigma^{2} S^{2} \frac{\partial^{2} u}{\partial S^{2}}-\frac{\partial u}{\partial \tau}-q \frac{\partial u}{\partial Q}+\mu S \frac{\partial u}{\partial S}-c u+g=0
$$

and holds throughout our chosen solution domain $H$. This will be subject to the specific boundary conditions which relate to the abandonment surface, far field conditions, expiry and exhaustion of the mine. We shall refer to (2.7) as the modified Feynman-Kac equation.

\section{(a) Probability of project completion}

We first show how equation (2.7) can describe the probability of completion. In this case we set $u=P$ in (2.7). In this instance we are not dealing with a monetary quantity, so therefore there is no discounting present, and so $c=0$. Next, we must consider the prescription of $f$, which is the limit of $P$ on the boundary. The probability of completion is evidently zero at abandonment, $f\left(S=S^{*}\right)=0$. At expiry, exhaustion and in the far field $(S \rightarrow \infty)$, completion is guaranteed, implying $f=1$ on these portions of the boundary. Finally, we may set $g=0$ as we do not consider a source term: we are simply interested in the expected value of $f$.

Making the above substitutions in (2.7) we find that the probability of completion is determined by the PDE

$$
\frac{1}{2} \sigma^{2} S^{2} \frac{\partial^{2} P}{\partial S^{2}}-\frac{\partial P}{\partial \tau}-q \frac{\partial P}{\partial Q}+\mu S \frac{\partial P}{\partial S}=0
$$

subject to

$$
\begin{gathered}
P=0 \quad \text { on } \quad S=S^{*}, \\
P=1 \quad \text { when } \min \{Q, \tau\}=0, \\
P \rightarrow 1 \text { as } S \rightarrow \infty .
\end{gathered}
$$

\section{(b) Expected lifetime}

We now set $u=D$ in equation (2.7) corresponding the expected lifetime of extraction. Since $D$ is also a non-monetary quantity, and there are no source terms present, again we have $c=0$ and $g=0$. However, the value of $f$ (which is the limit of the expected lifetime $D$, on the boundary) must be equal to the present time, $t$, and for large values of price the probability of abandonment becomes negligible, and so the mine will extract until expiry. These mean that equation (2.7) becomes

$$
\frac{1}{2} \sigma^{2} S^{2} \frac{\partial^{2} D}{\partial S^{2}}-\frac{\partial D}{\partial \tau}-q \frac{\partial D}{\partial Q}+\mu S \frac{\partial D}{\partial S}=0
$$

subject to the boundary conditions

$$
\begin{gathered}
D=T-\tau \text { when } \min \left\{S-S^{*}, Q\right\}=0, \\
D=T \text { when } \tau=0, \\
D \rightarrow T \text { as } S \rightarrow \infty .
\end{gathered}
$$




\section{(c) Mine valuation}

For completeness, we finally recover the equation for mine valuation. In this instance we set $u=V$ into (2.7), apply discounting equal to the interest rate, $c=r$, and because the mine will be worthless at abandonment, exhaustion and expiry, $f$ (the limit of $V$ on the boundary) will be zero there. The far field boundary condition is obtained by noting that the extraction rate will have a physical upper bound, and therefore the extraction costs are also bounded, and so the valuation will grow linearly in $S$ for large $S$. Finally we must now include the economic source terms generated during extraction. The costs incurred come in the form of extraction costs, $\epsilon_{M}$, and processing costs for refining the ore-bearing material, $\epsilon_{P}$, and both these terms may be functions of $Q$ (indirectly functions of time $t$ ), or even $S$, allowing known future changes in extraction and processing costs to be incorporated into the costs. The revenues arise from the amount of saleable ore, $q G S$, where $G$ is the amount of ore obtained per unit of material extracted. As mentioned previously in $\S 1$ random variations in the ore grade $G$ have been justifiably suppressed in this model, so that it may be expressed as a known function of $Q$.

This means $g$ is equal to the instantaneous cashflow $q G(Q) S-\epsilon_{P}(Q)-\epsilon_{M}(Q)$, and thus the mine valuation equation is described by

$$
\frac{1}{2} \sigma^{2} S^{2} \frac{\partial^{2} V}{\partial S^{2}}-\frac{\partial V}{\partial \tau}-q \frac{\partial V}{\partial Q}+\mu S \frac{\partial V}{\partial S}-r V+q G S-\epsilon_{P}-\epsilon_{M}=0,
$$

subject to the conditions

$$
\begin{gathered}
V=0 \quad \text { when } \quad \min \left\{S-S^{*}, \tau, Q\right\}=0, \\
V \sim S \quad \text { as } \quad S \rightarrow \infty .
\end{gathered}
$$

This equation is of the standard form derived for mining by Brennan \& Schwartz (1985) and derived for optimal gas storage by Chen \& Forsyth (2007).

\section{(d) Actual drift, or risk-neutral drift?}

The mine valuation obtained in part $(c)$ above is the expected net present value of the mine. Of course, this valuation is only correct under the risk-neutral measure. Indeed, an important consequence of a contingent claims-derived valuation is that the real-world percentage drift of the commodity price is not present in the valuation equation. This is a standard consequence of the hedging approach, in which a portfolio is created that contains the mine and is short a specified number of commodity contracts. The valuation equation thus obtained is

$$
\frac{1}{2} \sigma^{2} S^{2} \frac{\partial^{2} V}{\partial S^{2}}-\frac{\partial V}{\partial \tau}-q \frac{\partial V}{\partial Q}+(r-\delta) S \frac{\partial V}{\partial S}-r V+q G S-\epsilon_{P}-\epsilon_{M}=0,
$$

where $\delta$ is the convenience yield, which reflects transportation and storage costs (amongst other things) of the commodity. This relates to (2.8), (2.10) and (2.12) as follows. Under the risk-neutral measure, the price dynamics are given by

$$
d S=(r-\delta) S d t+\sigma S d X .
$$

Therefore, setting $\mu=r-\delta$ in equation (2.12) restores the PDE (2.14), and we make the same substitution to equations (2.8) and (2.10). We emphasise that this 
adjustment to the drift is well accepted and used throughout the literature, e.g. Hull (1993) and Hilliard \& Reis (1998). However, if hedging can not be performed, then the real-world percentage price drift is used for $\mu$ in (2.8), (2.10) and (2.12), and the solutions obtained are not risk-adjusted.

\section{Optimal position of abandonment}

To determine the surface of abandonment, $S^{*}=S^{*}(Q, t)$, we must find the position for which the valuation is indifferent between remaining operational or being abandoned. Whilst we acknowledge that there might be some penalty function incurred in abandoning (for example, those caused by terminating contracts early) which will alter the value, (McCarthy \& Monkhouse 2002), it is without loss of generality, and for clarity that we shall seek our abandonment position at $V=0$. As such, the option to abandon must satisfy the requirement

$$
V(S, Q, t) \geq \max (\bar{V}, 0),
$$

where $\bar{V}$ is the solution to the mine valuation (2.12) without the option to abandon. In other words, the value of the option to abandon is greater than the value of the regime which will always be extracting and the value of a mine which has been abandoned (which, in our case, is zero dollars). This is a similar approach to valuing American options, which are financial contracts which allow the owner to exercise at any point up till expiry (Wilmott et al. 1995). As such the problem can be expressed as a free-boundary problem, where the surface for where $V$ first becomes zero describes the free boundary in price for which it is optimal for the mining company to abandon. This problem is now nonlinear since $V$ depends on the free boundary, but the position of the free boundary is not known a priori. In order to determine this position precisely, one must do so during the calculation of the option using numerical techniques as described in $\S 5$.

As an alternative to using numerical techniques, a constant-valued approximation to $S^{*}$ enables a probability to be derived as a closed-form solution (see $\S 4$ ). To determine a suitable constant abandonment position one can use a price, $\hat{S}^{*}$ say, for which the mine valuation is approximately zero when one is not including the option to abandon. This approximate price to abandon will be greater than the actual abandonment price, because even though the lower actual abandonment price implies some negative cash flows can be incurred, this is justified by the probability that the price will rise again and the mine will regain profitability (Dixit \& Pindyck 1993). To give a clear methodology for this particular case of determining a constant valued abandonment price approximation, $\hat{S}^{*}$, we average each of our input functions over the interval $\left[0, Q_{\max }\right]$ making them constant, which also has the convenient effect of removing $Q$ from the model. This removal occurs because the extraction rate is now a constant, and therefore the size of the resource at any point in time is known a priori (see equation (2.5)) which means the valuation we are interested in is now a function only of $S$ and $t$. If we assume a risk-adjusted price process (2.15), then this averaging of the functions (and subsequent removal of the $Q$ derivative) reduces equation (2.12) to

$$
\frac{1}{2} \sigma^{2} S^{2} \frac{\partial^{2} V}{\partial S^{2}}-\frac{\partial V}{\partial \tau}+(r-\delta) S \frac{\partial V}{\partial S}-r V+\bar{q} \bar{G} S-\bar{\epsilon}_{P}-\bar{\epsilon}_{M}=0
$$


where the over-bars denote an averaged value for a function, and when coupled with the boundary conditions the solution is given by,

$$
V=\frac{S \bar{q} \bar{G}}{\delta}\left(1-e^{-\delta \tau}\right)-\frac{\bar{\epsilon}_{P}+\bar{\epsilon}_{M}}{r}\left(1-e^{-r \tau}\right) .
$$

The omission of $\sigma$ from this equation is expected, since the absence of the option to abandon means the cashflows of the mine can be replicated in advance via the purchase of forward contracts on the particular commodity (Brennan \& Schwartz 1985). An approximation to $\hat{S}$, which can easily be implemented, is applicable when either $r \tau$ and $\delta \tau$ and both are large, or when $r \approx \delta$, and is given by,

$$
\hat{S}=\frac{\delta\left(\bar{\epsilon}_{P}+\bar{\epsilon}_{M}\right)}{r \bar{q} \bar{G}} .
$$

\section{Closed-form solution}

To highlight a particular example of this model, which acts as a convenient approximation to the exact probability of completion, we shall derive a closed-form solution under two simplifying, but reasonable, assumptions. These are that the position of abandonment, $S^{*}$, is fixed (as discussed in $\S 3$ ), and the extraction rate, $q$, is a known function of time up until abandonment. This second simplification implies that $Q$ is also a specified function of time until abandonment, and we therefore do not need to consider $Q$ as an independent variable when solving away from the abandonment barrier (as was argued in the derivation of (3.2)). In making these two simplifications we are, in effect, collapsing the problem to a much simpler one, namely: what is the probability of a particle obeying the motion of (2.15) hitting a level barrier by a certain time? Since this particle problem has a well-known solution, these simplifications act as a methodology check on numerical solutions.

With these assumptions we may seek a solution to (2.8) of the form,

$$
P=1+\hat{P}(\tau, S),
$$

which, when coupled with a substitution for $S$ of the form

$$
Y=\log \left(\frac{S}{S^{*}}\right)
$$

transforms (2.8) into

$$
\frac{1}{2} \sigma^{2} \frac{\partial^{2} \hat{P}}{\partial Y^{2}}-\frac{\partial \hat{P}}{\partial \tau}+\left(r-\delta-\frac{1}{2} \sigma^{2}\right) \frac{\partial \hat{P}}{\partial Y}=0 .
$$

This is subject to the transformed boundary conditions,

$$
\begin{gathered}
\hat{P}=-1 \quad \text { on } \quad Y=0, \\
\hat{P} \rightarrow 0 \quad \text { as } \quad Y \rightarrow \infty, \\
\hat{P}=0 \text { on } \quad \tau=0 .
\end{gathered}
$$

The form of this equation allows us to reduce it to the well-known heat equation. We achieve this by making the transformation,

$$
\hat{P}=e^{\alpha Y+\beta \tau} g(Y, \tau),
$$


and setting

$$
\alpha=\frac{1}{2}-\frac{(r-\delta)}{\sigma^{2}}, \quad \beta=-\frac{\sigma^{2}}{2}\left(\frac{1}{2}-\frac{(r-\delta)}{\sigma^{2}}\right)^{2}
$$

which removes terms in $g$ and $\frac{\partial g}{\partial Y}$ respectively, allowing us to write

$$
\frac{1}{2} \sigma^{2} \frac{\partial^{2} g}{\partial Y^{2}}=\frac{\partial g}{\partial \tau}
$$

subject to,

$$
\begin{array}{r}
g=-e^{-\beta \tau} \quad \text { on } \quad Y=0, \\
g \rightarrow 0 \quad \text { as } \quad Y \rightarrow \infty \\
g=0 \text { on } \quad \tau=0 .
\end{array}
$$

This equation is simply the heat equation subject to homogeneous initial conditions and a non-homogeneous Dirichlet boundary condition. As such, we can now use the standard result (Cannon 1984) to write,

$$
g(Y, T)=-\int_{0}^{T} \frac{Y}{2 \pi \sigma^{2}(T-z)^{3}} \exp \left(-\frac{Y^{2}}{2 \sigma^{2}(T-z)}-\beta z\right) d z .
$$

This exact solution to (4.7), means that we can therefore write the probability of the mine remaining open until expiry as,

$$
P=1+e^{\alpha Y+\beta T} g\left(\log \left(\frac{S}{S^{*}}\right), T\right)
$$

where $g$ is given by (4.9). This can more conveniently be written as,

$$
P=1-\int_{0}^{T} \frac{\log (S)-\log \left(S^{*}\right)}{2 \pi \sigma^{2} z^{3}} \exp \left(-\frac{\left(\log (S)-\log \left(S^{*}\right)-\sigma^{2} \alpha z\right)^{2}}{2 \sigma^{2} z}\right) d z
$$

and is a known result from first passage problems, typically solved using probabilistic methods (Rogers \& Williams 2000).

A similar approach can be taken towards deriving the expected lifetime, $D$, from equation (2.10). This time we seek a solution of the form,

$$
D=T+\hat{D}(\tau, S)
$$

and then follow exactly the same approach as previously, to derive the result,

$$
D=T-\int_{0}^{T} \frac{\left(\log (S)-\log \left(S^{*}\right)\right)(T-z)}{2 \pi \sigma^{2} z^{3}} \exp \left(-\frac{\left(\log (S)-\log \left(S^{*}\right)-\sigma^{2} \alpha z\right)^{2}}{2 \sigma^{2} z}\right) d z .
$$

With these exact forms now derived, we can compare their behaviours with those of the numerically derived solutions which can handle a variable surface of abandonment. 


\section{Numerical method}

Before one can numerically calculate the probability of completion or the expected lifetime, one must first determine the optimal abandonment surface $S^{*}$, which is calculated from the mine valuation equation (2.14). When Brennan \& Schwartz (1985) first solved for mine valuation, they did so using a standard implicit finitedifference scheme. This type of numerical scheme is fine when there is no derivative in $Q$ present. However, such a scheme for solving (2.12), (2.8) and (2.10) can often exhibit complications which arise from potential discontinuities across the characteristic given by (2.5). These can cause large errors to permeate through the system, unless grid nodes are placed so that they coincide with the most important (characteristic) lines in the problem. To counter this, a recent and more suitable numerical scheme, known as the semi-Lagrangian method, has been used for solving systems similar to (2.12), e.g. Chen \& Forsyth (2007) and Evatt et al. (2010), and is equally applicable to (2.8) and (2.10). The advantage this scheme has over a regular implicit scheme, is that it (in effect) solves along the characteristic (2.5), thus removing issues arising from potential discontinuities (Falcone \& Ferretti 2002; Chen $\&$ Forsyth 2009). It cannot however remove them completely and there will still exist some numerical diffusion in $Q$, so alternative methods such as those described as total variation diminishing (TVD) may also be appropriate. However we find the semi-Lagrangian approach to be robust and accurate enough for our needs. For a general discussion on first exit times of multi-dimensional Ito diffusions - which do not exhibit discontinuities - see Patie \& Winter (2007).

Let us describe how the semi-Lagrangian scheme can be implemented to solve (2.8), (2.10) and (2.12). Consider the path (the semi-Lagrangian trajectory) through the domain $Q \times \tau$ which satisfies the equation,

$$
\frac{d Q}{d \tau}=q_{\max }
$$

Along this path the mine is always extracting at its maximum rate, $q=q_{\max }$. Using the initial condition that $Q(\tau=0)=0$, this characteristic line can be expressed as $Q=q_{\max } \tau$. Since the extraction rate is bounded by $q_{\max }$, we can say that in the region above this line $\left(Q>q_{\max } \tau\right)$ the mine cannot be emptied before the contract expires, so that trajectories in this region are no longer functions of $Q$, only of $\tau$. We therefore choose the grid spacing $\Delta Q=q_{\max } \Delta \tau$, so that there is always a node on the line $Q=q_{\max } \tau$ which separates the two regions.

In order to simplify the notation in the discretized equations, we write equation $(2.7)$ as

$$
\frac{\partial u}{\partial \tau}+q \frac{\partial u}{\partial Q}=\mathcal{L}\{u\}+g(S, Q, \tau),
$$

where $g$ is defined in $\S 2$ and the operator $\mathcal{L}$ is given by,

$$
\mathcal{L}\{u\} \equiv \frac{1}{2} \sigma^{2} S^{2} \frac{\partial^{2} u}{\partial S^{2}}+\mu S \frac{\partial u}{\partial S}-c u .
$$

Now consider a path through $Q \times \tau$ which satisfies the equation,

$$
\frac{d Q}{d \tau}=q
$$


then we define the left-hand-side of (5.2) as,

$$
\frac{D u}{D \tau}=\frac{\partial u}{\partial \tau}+\frac{d Q}{d \tau} \frac{\partial u}{\partial Q}
$$

We can ignore characteristics in $S$ as they have only second order effects. Hence the governing equation becomes

$$
\frac{D u}{D \tau}=\mathcal{L}\{u\}+g(S, Q, \tau)
$$

To keep matters simple we choose to discretize the system with an equally spaced grid in $S, Q$ and $\tau$ so that $S_{i}=i \Delta S, Q_{j}=j \Delta Q$ and $\tau_{k}=k \Delta \tau$. While the right-hand-side of equation (5.6) can be expressed with standard second-order finite differences at nodes in the grid, the left-hand-side may in general take values not located on a standard grid node, so that values on the latter will require interpolation. If we assume a fully implicit scheme discretizing at the node $(i, j, k)$ with $u_{i, j}^{k}=u\left(S_{i}, Q_{j}, \tau_{k}\right)$ and $q_{i, j}^{k}=q\left(S_{i}, Q_{j}, \tau_{k}\right)$, then in order to evaluate the total derivative on the left-hand-side we need to find the path in $Q$ that arrives at the point $Q_{j}$ at time $\tau_{k}$. The equation of the characteristic line (assuming $q_{i, j}^{k}$ is constant across $\left.\tau \in\left(\tau_{k-1}, \tau_{k}\right)\right)$ that satisfies this condition may be expressed as,

$$
\mathcal{Q}_{i, j}(\tau)=Q_{j}+q_{i, j}^{k}\left(\tau-\tau_{k}\right)
$$

enabling us to discretize the derivative on the left-hand-side to read

$$
\frac{D u}{D \tau}=\frac{u_{i, j}^{k}-u\left(S_{i}, \mathcal{Q}_{i, j}^{k-1}, \tau_{k-1}\right)}{\Delta \tau} .
$$

If the point $\left(S_{i}, \mathcal{Q}_{i, j}^{k-1}, \tau_{k-1}\right)$ does not coincide with a node, then linear interpolation is used to find the value $u\left(S_{i}, \mathcal{Q}_{i, j}^{k-1}, \tau_{k-1}\right)$. The full numerical scheme to solving (2.8), (2.10) and (2.12) may be written as

$$
\frac{u_{i, j}^{k}-u\left(S_{i}, \mathcal{Q}_{i, j}^{k-1}, \tau_{k-1}\right)}{\Delta \tau}=\mathcal{L}\left\{u_{i, j}^{k}\right\}+g\left(S_{i}, Q_{j}, \tau_{k}\right) .
$$

If higher accuracy is required, Crank-Nicolson timestepping will yield a second-order scheme, though care must be taken to difference the scheme at the half timestep along the characteristic in order to maintain second-order convergence.

In the case of the mine valuation, where the option to abandon is included, we have a nonlinear free-boundary problem which can be expressed as a constrained matrix problem with,

$$
V_{i, j}^{k} \geq 0
$$

The constrained matrix problem can be solved using a Projected Successive Over Relaxation (PSOR) method which is a standard numerical approach used on Americanstyle financial options (Wilmott et al. 1995). Once the valuation has been derived, the determination of $S^{*}(Q, t)$ is more straightforward: it is simply the root of $V\left(S^{*}, Q, t\right)=0$ (i.e. where the valuation is worthless). If there is a one-off penalty cost associated with abandonment, $K$, say, then $S^{*}$ would satisfy $V\left(S^{*}, Q, t\right)=-K$. 


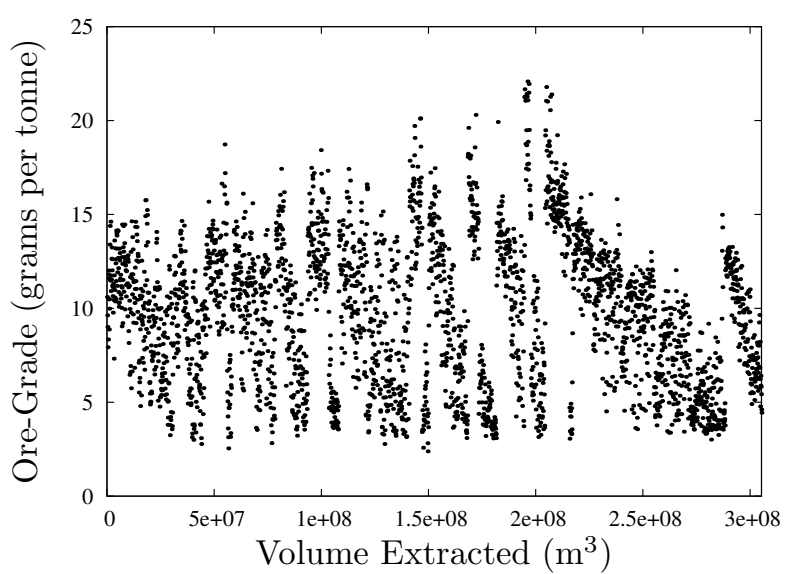

Figure 1. The estimated ore-grade variation of a mine along the extraction path of the mine, as a function of time. The ore-grade data was supplied by Gemcom Software International.

\section{Application to a real mine}

We now apply our model to a real mine data set, originally composed of some 30,000 data points, which has been provided by Gemcom Software International (a large mining solutions provider). The annual processing capacity is set at 20,000,000 orebearing tonnes per year, the cost of extraction, $\epsilon_{M}$, is set at $\$ 1$ per tonne, and the processing cost, $\epsilon_{P}$, is $\$ 4$ per tonne. Figure 1 shows how the ore-grade, $G$, varies throughout the path of extraction, having an average level of 9.74 grams per tonne. Numerically, when more than one piece of measured ore-grade data lies between two solution nodes of $Q$, it is the average value of those particular ore-grades which is used in the region around that point in $Q$. As such, one may think of our use of the ore-grade data as a localised moving average as one passes along the path of extraction. The final parameter values we use are given by,

$$
r=10 \% \mathrm{yr}^{-1}, \quad \delta=10 \% \mathrm{yr}^{-1}, \quad \sigma=30 \% \mathrm{yr}^{-\frac{1}{2}} .
$$

With the average ore-grade level we can use equation (3.4) to calculate an approximate constant abandonment price to be $\$ 0.51$ per gram, which - as explained in $\S 3$ - will typically be larger than the optimal level of abandonment.

\section{(a) One-dimensional abandonment price}

We first consider a relatively basic case where the mine will extract at a constant rate of 20,000,000 ore-bearing tonnes per year, which gives a maximum possible lifetime of 15.3 years. The resulting abandonment level, $S^{*}$, is shown in figure 2, which has been calculated numerically (see $§ 5$ ). The reason why this figure is one dimensional, as opposed to a surface, is that in this particular mine example the processing rate is fixed, and therefore the size of $Q$, is a known value which can be determined a priori at all points in time prior to abandonment, and therefore $S^{*}$ only depends on time. As can clearly be seen, this particular solution of $S^{*}$ exhibits quite a bit of variation, and we expect to see this passed into our solutions when looking at prices near the abandonment surface. 


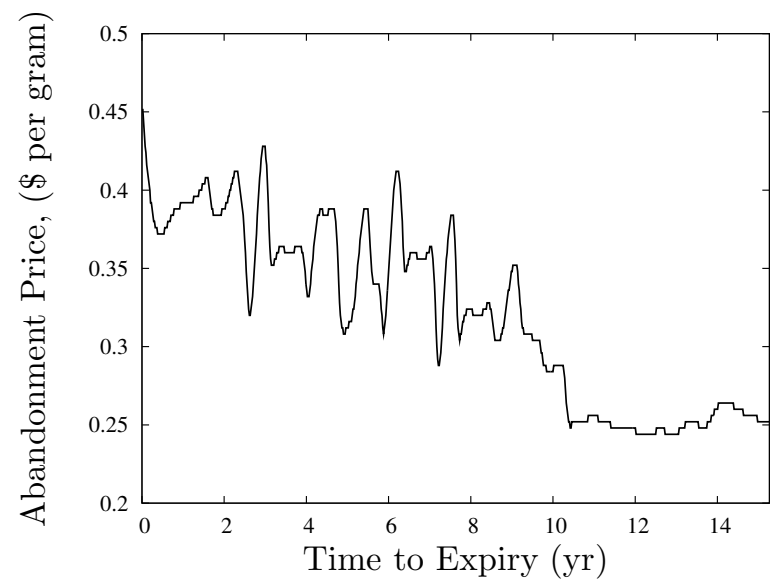

Figure 2. The optimal abandonment price during extraction where the rate of extraction is held at a constant level, calculated using the ore-grade data of figure 1 and the parameter values in (6.1).

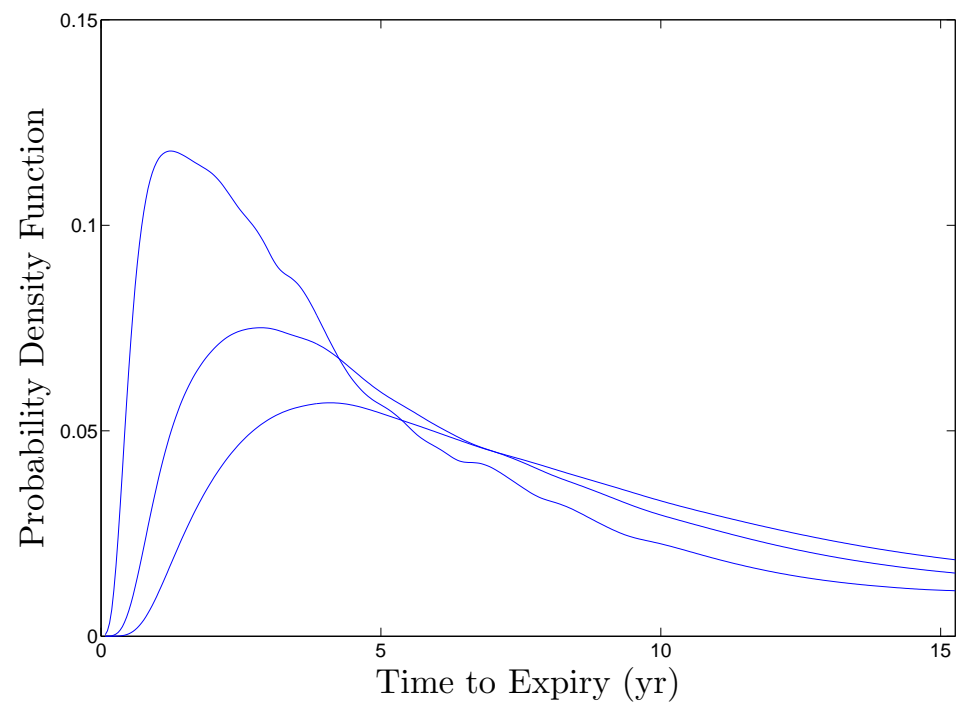

Figure 3. The probability density function of the probability of completion $P$, for three different prices: from highest peak to lowest peak, $S=0.8,1$ and 1.2. This was solved using numerical methods upon the data set shown in figure 1 and the parameters of (6.1). For smaller values of price, a larger degree of roughness is passed from the highly oscillatory abandonment surface, figure 2, to the solution of the PDFs shown here.

Using the numerically derived abandonment surface given by figure 2 , we start by calculating the probability density function (PDF) for $P$, figure 3 ; we do this for three underlying prices, $S=0.8, S=1$ and $S=1.2$. As can be seen, the closeness of the commodity price, $S$, to the abandonment price is reflected in the degree of variation of the $\mathrm{PDF}$, as the roughness of the abandonment surface has not yet 


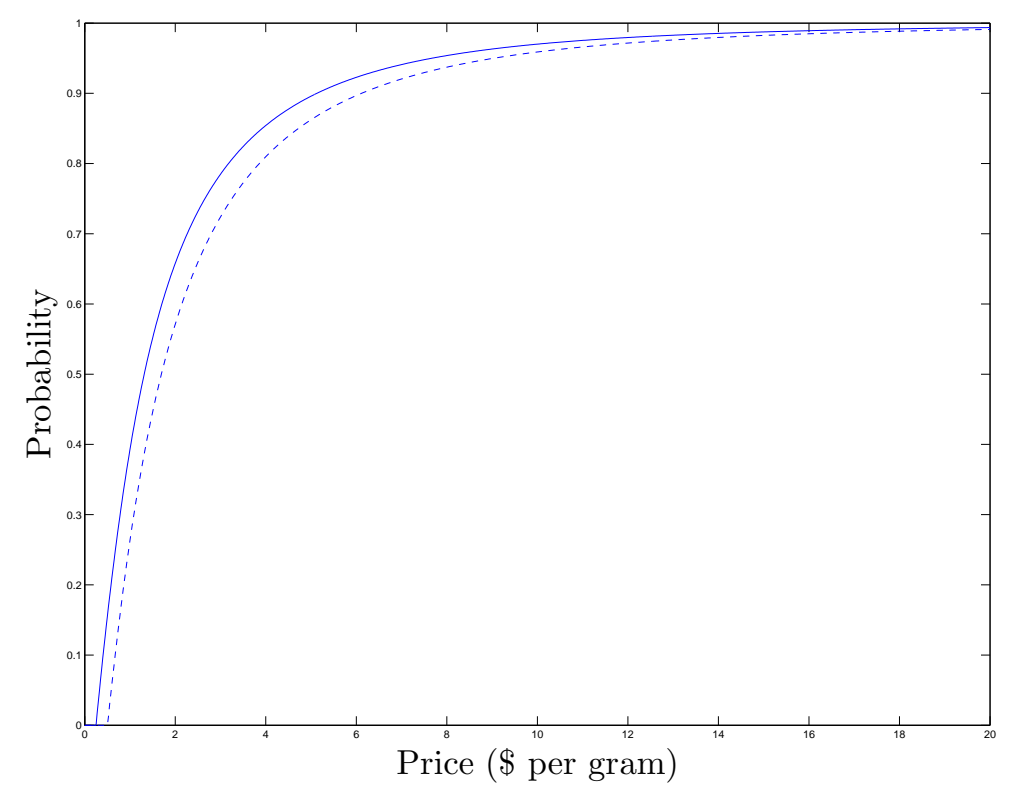

Figure 4. A comparison of probabilities of completion, $P$. The dashed line is the approximation (4.11), which assumes the position of abandonment is fixed for all time, $S^{*}=0.51$, and the continuous line is the solution to the full model (2.8) solved numerically using the real grade variation as shown in figure 1 . The remaining parameter values are that of (6.1)

negligibly diffused out for smaller price values. In addition, the lower the price is, the shorter the modal time to expiry (maxima of PDFs) becomes, as one would expect.

The probability of completion is shown in figures 4 and 5, where the absence of the $Q$ derivative enables us to use a regular implicit finite difference scheme (see section $§ 5$ ). As can be seen from figure 4 (continuous line), the solution undergoes a rapid period of change up to (approximately) $\$ 4$ per gram, and thereafter has a $85 \%$ probability of completion. Figure 5 shows how the probability of remaining open till expiry varies during extraction. This is shown for three different underlying prices, bottom to top, $S=0.8, S=1$ and $S=1.2$. The figure shows that for higher prices, the probability through time exhibits less curvature and approximately follows a near linear path between the initial probability, and $P=1$ at expiry.

Figure 4 also shows how the real-data numerical result (continuous line) compares to the closed-form solution, equation (4.11), which assumes a constant abandonment price (dashed line) over the remaining life of the mine. The two solutions exhibit a similar behaviour, where the key difference is a consequence of how the initial position of abandonment, $S^{*}(t=0)$, compares to that of the averaged position, $\hat{S}$. This is indeed a limiting factor for our approximation, since if the two positions were the same, the exact and approximate solutions would lie at the same point.

We now investigate the sensitivity of the probability of completion to the key 


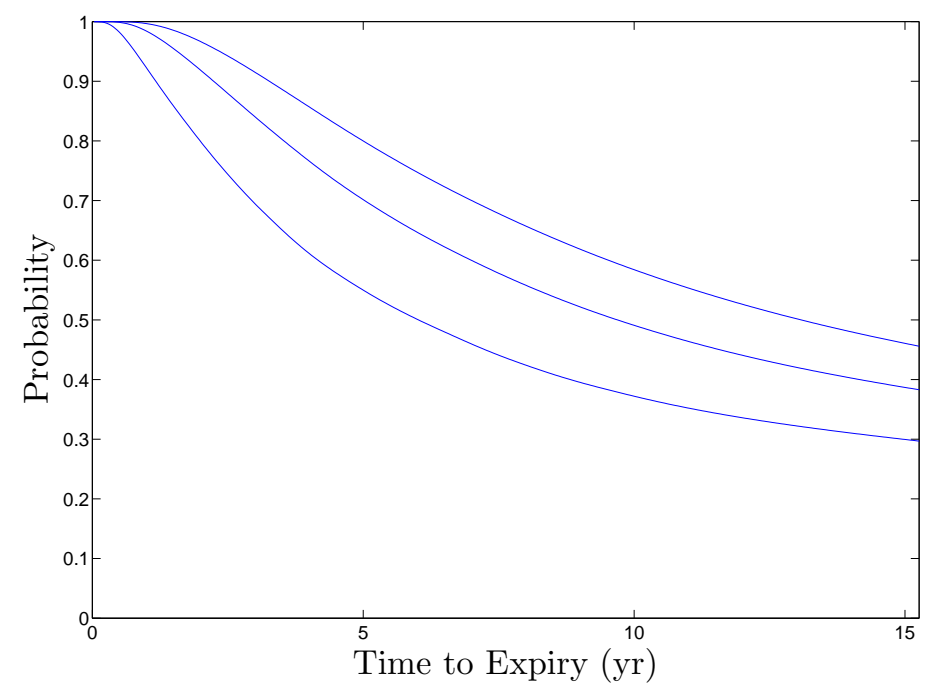

Figure 5. The probability of completion, $P$, as the operation progresses through time. The three solutions correspond to three different prices, $S=0.8,1$ and 1.2. These were solved using numerical methods, the data sets shown in figure 1 and the parameters of (6.1).

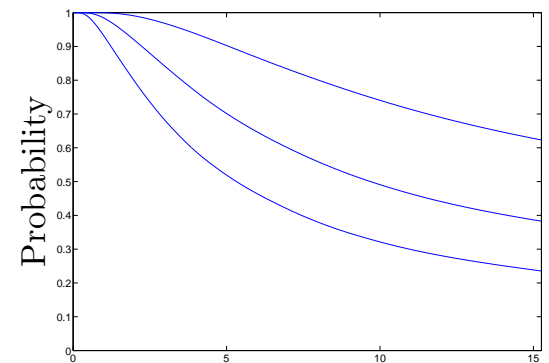

Time to Expiry (yr)

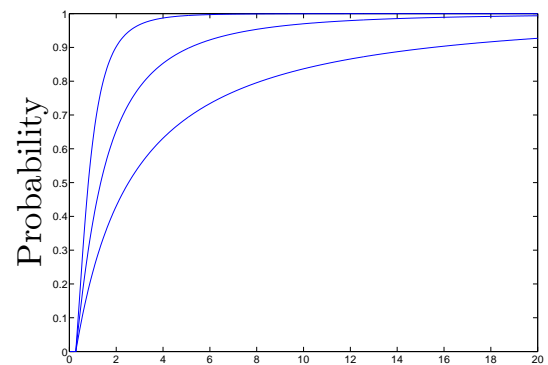

Price $(\$$ per gram)

Figure 6 . The sensitivity of the probability of completion, $P$, to price volatility, $\sigma$. From top to bottom, $\sigma=20 \%, 30 \%$ and $40 \%$. These were solved using numerical methods, the data sets shown in figure 1 and all other parameters are kept as those in (6.1).

underlying parameters, $\sigma$ and $r-\delta$. Figure 6 shows how the probability varies for three different values of $\sigma$, from bottom to top, $40 \%, 30 \%$ and $20 \%$. The right-hand graph shows the probability variation with price, and the left-hand graph shows variations of time to expiry, where an increase in volatility, obviously, increases the probability of closure. Although the forms of the solution remain similar, it is clear that even by varying the volatility by an absolute amount of $10 \%$, one can alter the probability quite strongly, where increases in $\sigma$ have a larger proportional effect than decreases. This is to be expected since, strictly speaking, the solution depends upon $\sigma^{2}$, not $\sigma$.

The sensitivity to the risk-adjusted drift, $r-\delta$, is shown in figure 7 . From bottom to top, the value of $r-\delta$ is $-5 \%, 0 \%$, and $5 \%$, where lower values of the risk-adjusted 


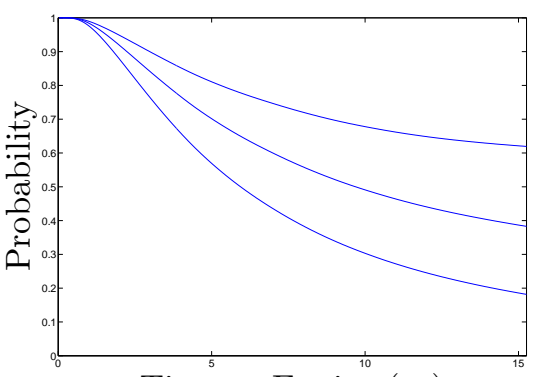

Time to Expiry (yr)

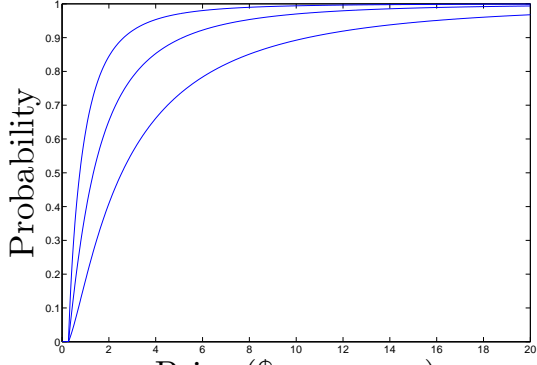

Price $(\$$ per gram)

Figure 7 . The sensitivity of the probability of completion, $P$, to the risk-adjusted drift rate, $r-\delta$. From top to bottom, $r-\delta=5 \%, 0 \%$, and $-5 \%$. These were solved using numerical methods, the data sets shown in figure 1 and all other parameters are kept as those in (6.1).

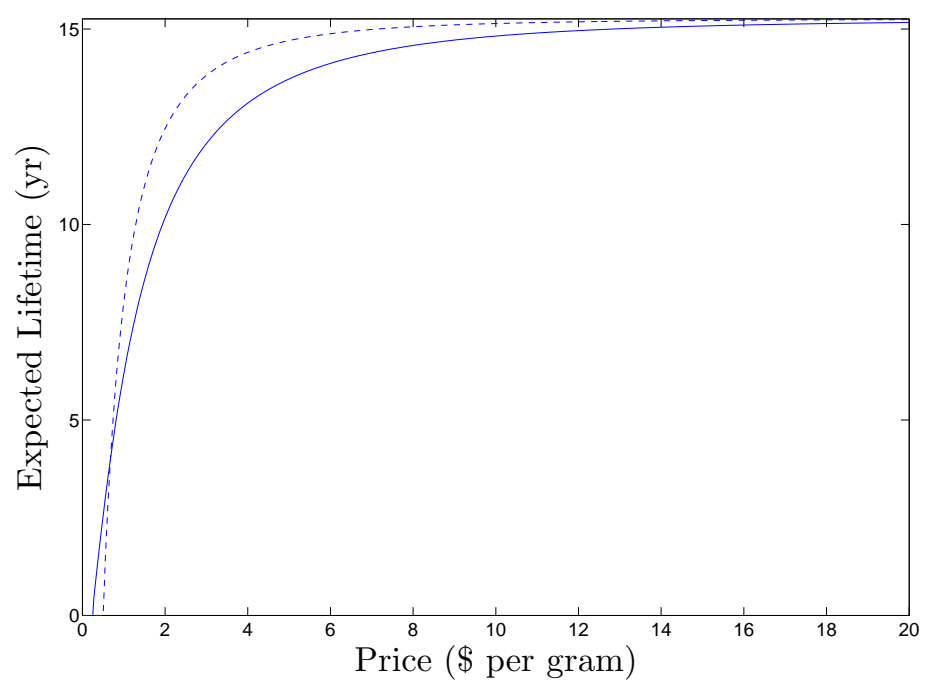

Figure 8. A comparison of expected lifetimes of the mine, $D$. The dashed line is the approximation (4.13) which assumes the position of abandonment is fixed, $S^{*}=0.51$, and the continuous line is the full solution to (2.10) which is solved numerically using the real grade variation as shown in figure 1. The parameters used are those of (6.1).

drift reduce the probability of remaining open. This makes intuitive sense, since if the risk-adjusted price was, on average, following a lower trajectory, we would expect the probability of it hitting the level of abandonment to be increased.

The expected time of closure (or first passage), is shown in figure 8. The continuous line is, once again, the numerical solution to (2.10), and the dashed line is the approximation under the assumption of a constant abandonment price. The results show a reasonable relationship between the two (albeit not as close as in the probability case), with the approximation having a greater curvature. The expected life of the mine exceeds 10 years for underlying prices over (roughly) $\$ 2$ per gram. 
(b) Two-dimensional abandonment price

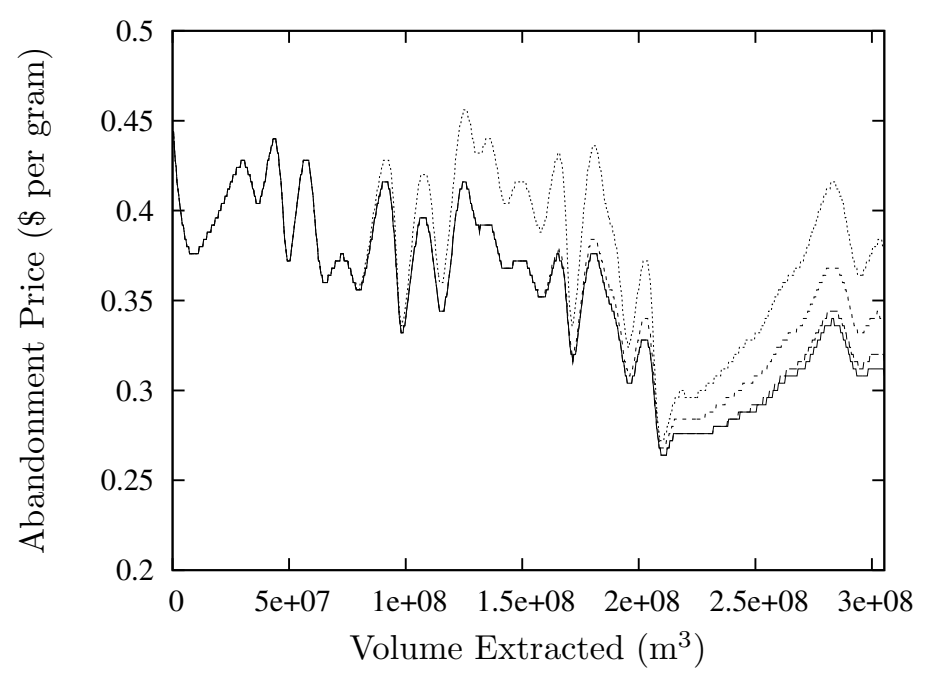

Figure 9. The optimal abandonment price during extraction where the extraction rate varies during extraction. Calculated using the ore-grade data of figure 1 and parameter values in (6.1). From highest to lowest, the four lines refer to $t=0,0.25 T, 0.5 T$ and $T$.

We next apply our model to the same mine, but with a more complex extraction regime. This particular regime has been created so as to optimise the decision to process ore-bearing tonnage or not (Johnson et. al. 2010), known as cut-off grade optimisation. Whilst the cut-off grade is not the focus of this paper, it does provide a convenient example where the resulting optimal abandonment surface is twodimensional, thus requiring the full array of numerical skills from $\S 5$. Figure 9 shows the optimal abandonment price throughout the mine, at each quartile of the maximum duration of extraction, $t=0,0.25 T, 0.5 T$ and $T$, where $T$ is now 14.1 years. This figure uses the same ore-grade data as that of figure 1 and the parameter values in (6.1).

By using the abandonment surface of figure 9 and the semi-Lagrangian method, we can now solve (2.8) and (2.10) to give the probability of completion, which is shown in figure 10 (left), and the expected lifetime of the mine, shown in figure 10 (right). The results are similar to those previously derived, where most change in the probability occurs over prices below (around) $\$ 3$ per gram, and above this price the expected lifetime of the mine remains close to the maximum possible duration.

\section{Conclusions}

This paper has presented a method for analysing the probability of an extraction project being completed in the presence of commodity price uncertainty. This problem is equivalent to a first-passage distribution, in which the project stops if the price reaches a certain boundary. The method utilises the Feynman-Kac approach to derive a governing PDE where the presence of a derivative in the resource size makes this problem distinct from regular first-passage problems. The PDE can be 

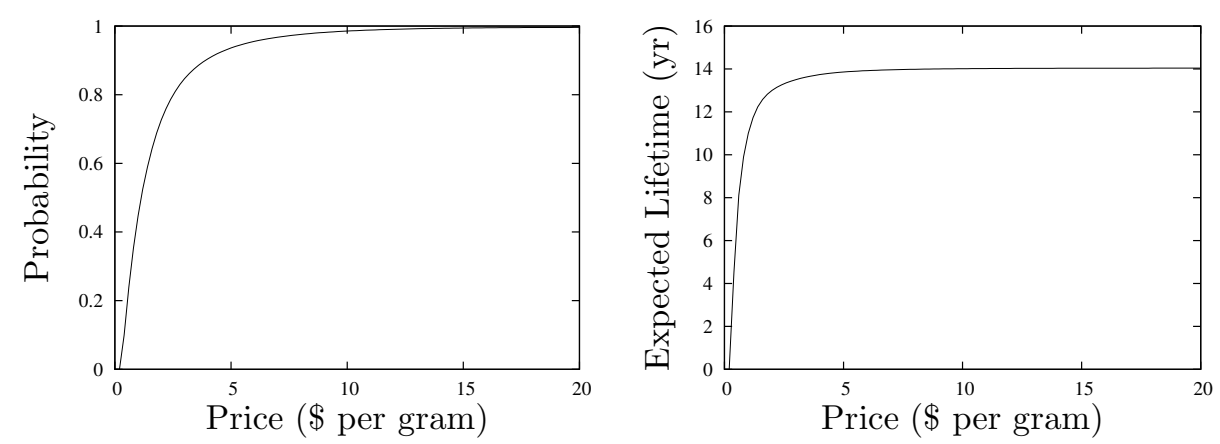

Figure 10. The left hand figure shows the probability of completion against price, and the right hand figure shows the expected lifetime of the mine. These were solved using the semi-Lagrangian method as detailed in $\S 5$, the data set shown in figure 1 , the abandonment surface shown in figure 9 , and the parameters in (6.1).

utilised to derive the probability of project completion, the expected lifetime of extraction and the mine valuation itself. We explain how to solve for these by the semi-Lagrangian numerical method, which overcomes the problem of potential discontinuities from the solutions. As an approximation to these numerical solutions, we derived closed-form solutions for the probability of completion and the expected time of stopping, under the simplifying assumption of a constant abandonment price over the life of the mine. The results show that our approximation yields a good resemblance to the solutions computed with real data.

Future work in this area may well focus upon the inclusion of a jump process in price (such as a Lévy process), since for some commodities this is arguably a more suitable representation (Hilliard \& Reis 1998). In addition, the probability of a specific event occurring can be calculated for a wide range of investor decisions using the methods developed here.

This project was aided by funding from the 'SPRIng' project of the Engineering and Physical Sciences Research Council, UK. The authors are grateful to Gemcom Software International who supplied the ore-grade data, and provided valuable advice on mining operations. The authors are also grateful to the two referees who helped make valuable suggestions.

\section{References}

Bessembinder, H., Coughenour, J.F., Seguin, P.J. \& Smoller, M.M. 1995 Mean Reversion in Equilibrium Asset Prices: Evidence from the Futures Term Structure, Journal of Finance, L, 1, 361-375.

Black, F. and Scholes, M. 1973 The Pricing of Option and Corporate Liabilities, Journal of Political Economy, 81, No. 3.

Black, F. 1976 The Pricing of Commodity Contracts, Journal of Financial Economics, 3, 167-179.

Brennan, M.J. \& Schwartz, E.S. 1985 Evaluating Natural Resource Investments, The Journal of Business, 58, 2, 135-157.

Caccetta, L. \& Hill, S.P. 2003 An Application of Branch and Cut to Open Pit Mine Scheduling, Journal of Global Optimisation, 27, 349-365.

Cannon, J., R. 1984 The One Dimensional heat Equation, Cambridge University Press. 
Cherian, J.A., Patel. J. \& Khripko, I. 2000 Optimal Extraction of Nonrenewable Resources When Costs Cumulate, Project Flexibility, Agency, and Competition, ed. M.J. Brennan, and L. Trigeorgis, Oxford University Press.

Chen, Z. \& Forsyth, P.A. 2007 A semi-Lagrangian Approach for Natural Gas Storage Valuation and Optimal Operation, Siam J. Sci. Comput., 30, 1, 339-368. (doi:10.1137/060672911)

Chen, Z. \& Forsyth, P.A. 2010 Implications of a Regime-Switching Model on Natural Gas Storage Valuation and Optimal Operation, Quant. Finance, 10, 2, 159-176. (doi:10.1080/14697680802374791)

Dixit, A.K. \& Pindyck, R.S. 1994 Investment under Uncertainty, Princeton University Press.

Evatt, G.W., Johnson, P.V., Duck, P.W. \& Howell, S.D. 2010 Mine Valuations in the Presence of a Stochastic Ore-Grade, accepted for publication by the International Association of Engineers.

Falcone, M. \& Ferretti, R. 2002 Convergence Analysis for a Class of High-0rder Semi-Lagrangian Advection Schemes SIAM J. Numer. Anal., 35, No.3, 909-940. (doi:10.1006/jcph.2001.6954)

Gibson, R. \& Schwartz, E.S. 1990 Stochastic Convenience Yield and the Pricing of Oil Contingent Claims, The Journal of Finance, XLV, 3, 959-976.

Heath, D., Jarrow, R. \& Morton A. 1992 Bond Pricing and the Term Structure of interest Rates: A New Methodology for Contingent Claims Valuation. Econometrica, 60, No.1, $77-105$.

Hilliard, J.E. \& Reis, J. 1998 Valuation of Commodity Futures and Options under Stochastic Convenience Yields, Interest Rates, and Jump Diffusions in the Spot, Journal of Financial and Quantitative Analysis, 33, 1, 61-86.

Hull, J.C. 1993 Options, Futures, and other Derivative Securities, 2nd edn. Prentice Hall.

Jewbali, A., \& Dimitrakopoulos, R. 2009 Stochastic Mine Planning - Example and Value from Integrating Long- and Short-Term Mine Planning Through Simulated Grade Control, Orebody Modelling and Strategic Mine Planning, 2nd edn. The Australasian Institute of Mining and Metallurgy, Melbourne, 327-333.

Johnson, P.V., Evatt, G.W., Duck, P.W. \& Howell, S.D. 2010 The Derivation and Impact of an Optimal Extraction Regime upon Mine Valuations, accepted for publication by the International Association of Engineers.

Kloeden, E. \& Platen, E., 1992 Numerical Solution of Stochastic Differential Equations, 6 th edn. Springer-Verlag.

Martinez, L.A. 2009 Designing, Planning and Evaluating a Gold Mine Project Under InSitu Metal Grade and Metal Price Uncertainties, Orebody Modelling and Strategic Mine Planning, The Australasian Institute of Mining and Metallurgy, Melbourne, second edition, 225-234.

McCarthy, J. \& Monkhouse, H. L. 2002 To Open Or Not To Open - Or What to Do With a Closed Copper Mine. Journal of Applied Corporate Finance, 15, 2.

Menabde, M., Foyland, G., Stone, P., \& Yeates, G.A. 2004 Mining Schedule Optimisation for Conditionally simulated Orebodies, Proceedings of the International Symposium on Orebody Modelling and Strategic Mine Planning: Uncertainty and Risk Management, $347-52$.

Øksendal, B. 2003 Stochastic Differential Equations, 5th edn, Springer.

Patie, P. \& Winter. C. 2008 First exit time probability for multidimensional diffusions: A PDE-based approach. Journal of Computational and Applied Mathematics, 222, 42-53. (doi:10.1016/j.cam.2007.10.043)

Rogers, L.C.G. \& Williams, D., 2000 Diffusions, Markov Processes and Martingales, 1, Cambridge University Press. 
Schwartz, E.S. 1997 The Stochastic Behaviour of Commodity Prices: Implications for Valuation and Hedging, The Journal of Finance, LII, 3, 923-973.

Trigeorgis, L. 1993 The Nature of Option Interactions and the Valuation of Investments with Multiple Real Options. Journal of Financial and Quantitative Analysis,28, No. 1, $1-20$.

Thompson, M., Davison, M. \& Rasmussen, H., 2004 Valuation and Optimal Operation of Electric Power Plants in Competitive Markets, Operations Research, 52, 4, 546-562.

Wilmott, P., Howison, S. \& Dewynne, J. 1995 The Mathematics of Financial Derivatives, Cambridge University Press.

Williams, D. 1991 Probability with Martingales, Cambridge University Press. 